LA W REN CE LIVERMORE N A T IO N A L LABORATORY

Position Estimation of Transceivers in Communication Networks

C.A. Kent, F.U. Dowla

January 23, 2004

Fifth IEEE Workshop on Signal Processing Advances in Wireless Communications Lisbon, Portugal July 11, 2004 through July 14, 2004 
This document was prepared as an account of work sponsored by an agency of the United States Government. Neither the United States Government nor the University of California nor any of their employees, makes any warranty, express or implied, or assumes any legal liability or responsibility for the accuracy, completeness, or usefulness of any information, apparatus, product, or process disclosed, or represents that its use would not infringe privately owned rights. Reference herein to any specific commercial product, process, or service by trade name, trademark, manufacturer, or otherwise, does not necessarily constitute or imply its endorsement, recommendation, or favoring by the United States Government or the University of California. The views and opinions of authors expressed herein do not necessarily state or reflect those of the United States Government or the University of California, and shall not be used for advertising or product endorsement purposes. 


\section{Position Estimation of Transceivers in Communication Networks}

\author{
Claudia A. Kent \\ Lawrence Livermore National Laboratory, L-229 \\ Livermore, CA 94550 USA \\ E-mail: kent12@1lnl.gov
}

\begin{abstract}
With rapid developments in wireless sensor networks, there is a growing need for transceiver position estimation independent of GPS, which may not be available in indoor networks. Our approach is to use range estimates from time-of-flight (TOF) measurements, a technique well suited to large bandwidth physical links, such as in ultrawideband (UWB) systems. In our UWB systems, pulse duration less than 200 psecs can easily be resolved to less than a foot. Assuming an encoded UWB physical layer, we first test positioning accuracy using simulations. We are interested in sensitivity to range errors and the required number of ranging nodes, and we show that in a highprecision environment, such as UWB, the optimal number of transmitters is four. Four transmitters with $\pm 20 \mathrm{ft}$. range error can locate a receiver to within one or two feet. We then implement these algorithms on an 802.11 wireless network and demonstrate the ability to locate a network access point to approximately 20 feet.
\end{abstract}

\section{INTRODUCTION}

$\mathrm{I}_{\mathrm{e}}^{\mathrm{n}}$ many sensor network applications, such as environmental monitoring of ground water or airborne chemicals, firefighters in buildings, or soldiers in caves, it is important to know the position of the network nodes. Range estimation from TOF data between communicating nodes is particularly attractive when using short-duration or high-frequency pulses such as UWB systems, and to a lesser extent for wireless local area network links in the 2.4 and $5 \mathrm{GHz}$ bands. For example, from radar theory, the root mean square (rms) range error in meters is given by [6]:

$$
\delta R \approx \frac{c}{B W \sqrt{S N R}}
$$

where BW is the bandwidth of the pulse, SNR is the signal to noise ratio at the receiver, and $\mathrm{c}$ is the speed of light, 3x108m/s. For bandwidths of $10 \mathrm{MHz}, 100 \mathrm{MHz}$, and $1 \mathrm{GHz}$ (corresponding approximately for $802.11 \mathrm{~b}$, 802.11a, and UWB systems), the rms range errors are $3 \mathrm{~m}, 0.3 \mathrm{~m}$ and $0.03 \mathrm{~m}$, respectively, for an assumed SNR of $20 \mathrm{~dB}$. We cannot expect to achieve this accuracy here, as we are using standard communication protocols and not dedicated radars, so we expect our range errors to increase one to two orders of magnitude. The range errors for an 802.11-a link can then be anywhere from 3

\author{
Farid U. Dowla \\ Lawrence Livermore National Laboratory, L-290 \\ Livermore, CA 94550 USA \\ E-mail: dowla1@1lnl.gov
}

to 30 meters. We expect the more robust UWB systems to perform better than this, the wideband nature of the pulses allows us to determine the arrival times in a correlation filter more precisely than in narrow band systems. For example, in UWB systems developed at LLNL, the radio-frequency (RF) pulse duration is only about 200 pico-seconds. Hence, the arrival time of the pulses can resolved to less than a foot.

In this work, we first assume a high-precision ranging mechanism such as UWB and we simulate position estimation for a set of communicating nodes. We next implement the technique on actual 802.11 hardware to test the capability in a low-precision environment. This paper is a discussion of our simulation investigation on high-precision node positioning from TOF data and our low-precision implementation on an 802.11 network.

For the high-precision simulations, a network consists of transmitter and receiver nodes distributed randomly in a $100 \mathrm{~m} \times 100 \mathrm{~m}$ area. Transmitters have known position via satellites or some other method, receivers have unknown position. Transmitters determine receiver position through time-of-flight ranging and information sharing. By simulating ranging in this scenario, we can describe the relationship between ranging accuracy and position estimation accuracy, the improvement in position estimation with additional transmitting nodes, and the benefit of using a "ranged" receiver node as a pseudo-transmitter. Interested readers are referred to our references for a more extensive survey of current research in this area.

In the 802.11 implementation we address the need for network security where an access point may be providing connectivity to unapproved users, transmitting unwanted data, or otherwise acting in a non-compliant manner, and we seek to locate its position. Experimental limitations require us to address only the inadvertent violator scenario. In a real world application, we could use system-level transactions allowing utility in a more hostile environment. Assuming all nodes communicate with each other via an access point, and the 802.11 signals propagate through walls, a range measurement between a node and an access point is proportional to their distance. The transaction we choose is the PING. 
The version distributed by the Microsoft Corp. measures time-of-flight in msecs; instead of this, we use a version where trip delay is given in $\mu$ secs, hrPING distributed by cFOS Corp. in Denmark.

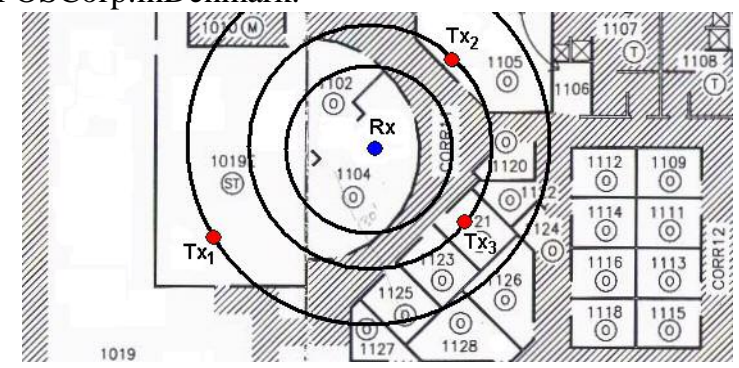

Figure 1. In the MATLAB GUI-based software the user designates a network of transmitters and receivers and simulates the network in ranging, filtering, data management, and position estimation.

\section{SOFTWARE INTERFACE}

We developed a MATLAB GUI-based communication and simulation package for two goals: to simulate virtual networks of transmitters and receivers where the user specifies the error associated with the ranging transactions, and to act as an interface on a real network of transmitters and receivers. Both goals require a ranging mechanism, a data sharing communications infrastructure, and position estimation algorithms. A screenshot of the interface is shown in Fig. 1. The software simulates (in the UWB case) or implements (in the 802.11 case) actual ranging, maintains the communications infrastructure, measurement filtering, and information sharing allowing position estimation.

Using the simulation environment, we quantified the relationship between transmitter ranging accuracy and receiver position estimation accuracy, the level of improvement with additional transmitters, and determined if a "located" receiver can act as a "pseudotransmitter" to improve the position estimate of other receivers. In the hardware environment we implemented the technique on a wireless 802.11 network to test the capability of ranging and positioning.

\section{A. Range Measurement Error}

An UWB TOF range measurement will include error from several sources. Neither signal multi-path, nor receiver processing time can be predicted precisely. We model this error as a uniformly distributed constant and assign to our simulated range measurements a random measurement-bias within ranges of $\pm 5 \mathrm{ft}$., $\pm 10 \mathrm{ft}$., etc.

The measurement-bias models the process error in a real system, and we assume, a filter used eliminate the measurement-bias would also eliminate process error. We continuously collect range measurements and filter them using a weighted least squares filter. It takes a set of measurements within a fixed-length time window in a linear model, and weights them according to their inverse variances. As each new measurement arrives, we calculate the new variance and find $\mathrm{R}^{*}$, our bias-free range estimate, from the most recent set of measurements within our time window.

In the 802.11 hardware environment, the ranging transaction PING is sub-optimal for several reasons. First, PING is a high-level protocol and a low-priority in the CPU stack; the $\mu$ secs spent doing "other things" reduces the accuracy of the time of flight measurement. Second, it requires full cooperation from the receiver, nullifying an obvious application to locate an "out of compliance" network node. If a node were maliciously out of compliance, we assume it will not respond to a PING request. We must then assume that a noncompliant node is acting unintentionally, and propose a future solution to both problems by replacing PING with a communication protocol on the physical-layer to solve CPU stack delays and potentially allowing communication in a non-cooperative environment.

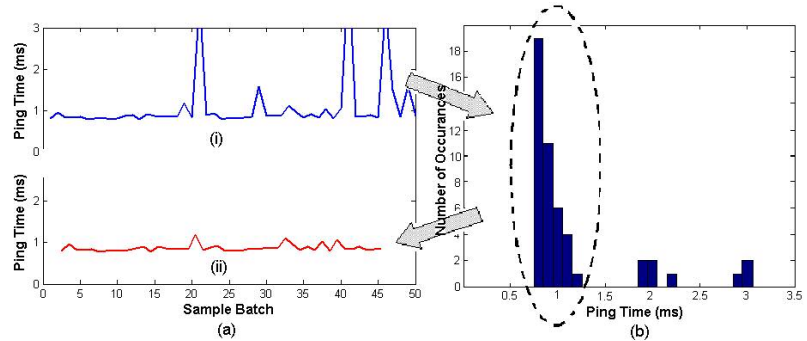

Fig. 2. The histogram filtering takes the noisy data in (a)(i) and removes the outliers, keeping only the first subset of data in (b). The results are in (a)(ii).

Every PING issued by a transmitter results in a batch of replies noting the elapsed time. As each batch arrives we send it through two stages of filtering to extract the real PING time. In the first filter stage, we distribute the data in a histogram of $100 \mu \mathrm{sec}$ width bins. The data in Fig. 2(a)(i) is shown in a Histogram in Fig. 2(b), where the primary subset, or "first hump" is extracted and replotted in Fig. 2(a)(ii). This stage removes the disproportionately large spikes in the data of Fig. 2(a)(i), leaving the data within a range of approximately 100$300 \mu \mathrm{s}$, as opposed to the original $5 \mathrm{~ms}$ range.

The second filter stage is a recursive weighted leastsquares estimator, chosen for it's ability to predict a the true value of a variable given sequential batches of "noisy" variable measurements over time. The filter works recursively by updating the least-squares solution after every new batch of data arrives. For the PING issued at the $k^{\text {th }}$ sampling interval, we receive a batch of $m$ new measurements $\mathbf{z}_{k}$, and we estimate the PING 
time at the next interval $z_{k+1}$, and call it $\hat{z}_{k+1}$. To achieve this, we assume $\mathbf{z}_{k}$ takes the form

$$
\mathbf{z}_{\mathbf{k}}=\mathbf{H x}_{\mathbf{k}}+\mathbf{n}_{\mathbf{k}} \quad \text { where } \quad \mathbf{H}=\left[\begin{array}{cc}
1 & t_{1} \\
\vdots & \vdots \\
1 & t
\end{array}\right]
$$

The matrix $\mathbf{H}$ defines the system type, we assume a firstorder system of constant velocity, and the vector $\mathbf{n}_{k}$ is the residual measurement error. If we knew the value of $\mathbf{x}_{k}$, we could solve for $\hat{z}_{k+1}$, the PING estimate at the next measurement. The WLS solution to (1) is

$$
\hat{\mathbf{x}}_{k}=\hat{\mathbf{x}}_{k-1}+\mathbf{K}_{k}\left(\mathbf{z}_{k}-\mathbf{H} \hat{\mathbf{x}}_{k-1}\right)
$$

which is the estimate of $\mathbf{x}_{k}$ that minimizes a quadratic cost function of residual error. A thorough derivation of (4) is found in [8]. The solution consists of the previous estimate plus the residual error scaled by a gain matrix. The gain matrix is

$$
\mathbf{K}_{k}=\mathbf{P}_{k-1} \mathbf{H}^{T}\left(\mathbf{H} \mathbf{P}_{k-1} \mathbf{H}^{T}+\mathbf{R}_{k}\right)^{-1}
$$

where $\mathbf{P}_{k}$ is the error covariance matrix representing the error after the $k$ th estimate.

$$
\mathbf{P}_{k}=\left(\mathbf{P}_{k-1}{ }^{-1}+\mathbf{H}^{T} \mathbf{R}_{k}^{-1} \mathbf{H}\right)^{-1}
$$

Finally, we presume some of our measurements are better than others, and we define a "weighting matrix" $\mathbf{R}_{k}$ proportional to each new measurement's variation from the previous estimate, or

$$
\mathbf{N}_{k}=\mathbf{I} \otimes \mathbf{z}_{k}-\mathbf{I} \hat{z}_{k-1} \quad \text { and } \quad \mathbf{R}_{k}^{-1}=\left(\mathbf{N}_{k}^{T} \mathbf{N}_{k}\right)^{-1}
$$

where the operator $\otimes$ is the element-by-element product of the measurement vector $\mathbf{z}_{k}$ with the identity matrix, resulting in a diagonal matrix of measurement values. The weights "reward" the samples that are more closely equal to the previous estimate in a feedback sense. This processing results in a single scalar new estimate of round-trip flight time, $\mathrm{R}^{*}$.

\section{B. Generating Position Estimates}

The MATLAB software maintains a communications infrastructure to allow the transmitters to share their most current WLS-filtered range estimates, $\mathrm{R}^{*}$, associated with each receiver. Recall the range estimate is simply the round-trip TOF filtered using the methods detailed in Sect. IIa and multiplied by the velocity of the signal (the speed of light). With enough $\mathrm{R}^{*}$ 's, a position estimate is calculated using the closedform method detailed in [7]. A graphical representation of the method is shown in Figure 3, where the $\mathrm{R}^{*}$ measurements from two transmitters are combined in the Pythagorean Theorem (PT) to find receiver position. We combine the known transmitter positions and the estimated receiver distances in multiple PT equations solved simultaneously to minimize equation error in a least-squares fashion. A minimum of three transmitters, and the corresponding three $\mathrm{R}^{*}$ measurements, is required for a unique receiver position. Two are shown in the figure, but a mirror triangle could be calculated placing a receiver alternate outside of the concentric circles, thus three transmitters eliminate ambiguity. It is important to note that a solution to the position estimation problem is possible even in the case of large range measurement error since the algorithm in [7] acts to find the least squares solution, or the one that results in the overall minimization of equation error.

All transmitters maintain the range measurement information between themselves and all receivers in the network. They share only the filtered range measurements with the other transmitters. Once a transmitter has range measurements between a receiver and three separate transmitters, it can independently calculate the receiver's position estimate using the technique in Fig. 3.

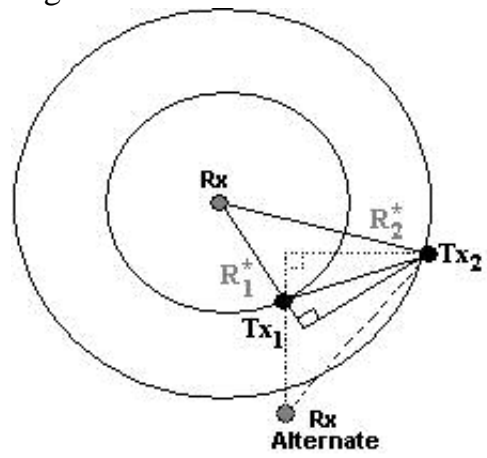

Figure 3. In this graphical representation of the closed-form least squares position estimation method developed in [7], the range measurements from multiple transmitters are combined using the Pythagorean Theorem for an estimate of position.

\section{RESULTS}

During a high-precision simulation, the position estimate of a receiver typically converges to and remains at a settled value after 1000 timesteps (one minute of sampling at 10ms). To insure convergence, we run all simulations for approximately 3000 timesteps. We generate hundreds of random networks for each experiment, and we take the final, converged value as the position error associated with the network.

\section{A. Ranging Accuracy and Additional Transmitters}

To measure the effect of additional transmitters on position error, we use 100 random networks of the minimum size, three transmitters and one receiver, and we simulate each with a small uniformly distributed range measurement error $( \pm 20 \mathrm{ft}$.). We then calculate the 
average and standard deviation of the converged values across all of the 100 networks and repeat the test while varying the number of transmitters from three through nine. The results are compiled in the errorbar plot of Fig. 4 with mean position error and standard deviation as a function of number of transmitters. By increasing the number of transmitters to four, mean position error decreases by nearly 20ft., and measurement confidence increases (with a standard deviation decrease) by nearly 60ft. Increasing the number of transmitters to five, however, shows little additional improvement. Four transmitters independently ranging a receiver with $\pm 20 \mathrm{ft}$. accuracy can locate its position within less than $5 \mathrm{ft}$.

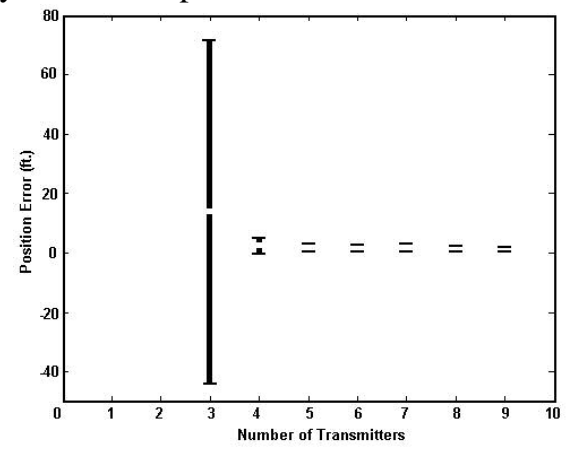

Figure 4. The mean and mean + STD were collected for networks ranging from three to nine transmitters and one receiver. All networks assumed a $\pm 20 \mathrm{ft}$. range measurement error. Four transmitters dramatically reduce both mean and standard deviation.

In Fig. 4 the range measurement error is centered between $\pm 20 \mathrm{ft}$., and four transmitters provide optimal position accuracy. In Fig. 5 we present data collected by varying range measurement error along with number of transmitters to find an overall correlation between the three. Confidence in four transmitters, rather than three, is valid only when range measurement error is kept below $\pm 60 \mathrm{ft}$. Above this, additional transmitters are necessary.

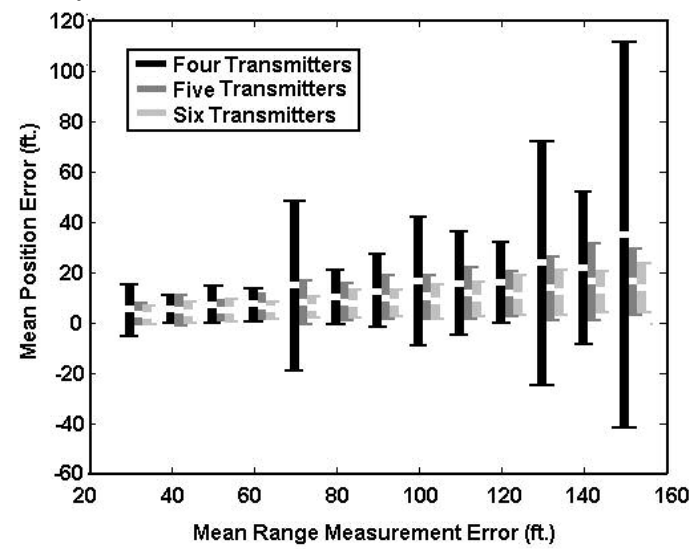

Figure 5. We are interested in the effect additional transmitters, but also the effect of an increased range measurement error. These errorbar plots of mean and standard deviation show the impact on position error by varying both of these factors with four transmitters, and one receiver, five transmitters and one receiver, and six transmitters and one receiver.

\section{B. Pseudo-Transmitters}

Once a receiver has been "located," we are interested in using it to improve the position estimate of another receiver and thus consider it a pseudo-transmitter. In this case, there is no difference between a transmitter and receiver (save the three dedicated transmitters needed for location and orientation reference). We test this idea using $N$ real transmitters and $M$ pseudo-transmitters, and we find that pseudo-transmitters do not improve the position estimate of a receiver as do real transmitters; instead, they introduce an undamped oscillation that worsens with additional pseudo-transmitters. We test this by varying $N=[3, \ldots, 6]$ and $M=[1, \ldots, 6]$ and find all cases similar to that shown in Fig. 6 where $N=5$ and M $=[1, \ldots, 4]$. As the number of pseudo-transmitters increases, so does position error. The pseudo-transmitter does add knowledge to the system, however with the slightest amount of error (here $\pm 10 \mathrm{ft}$.) the system becomes unstable.

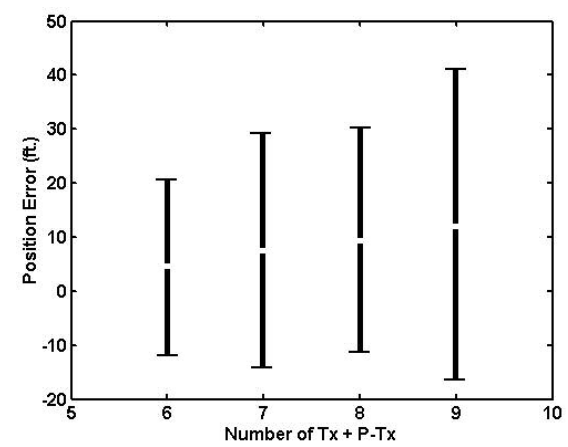

Figure 6: We use five transmitters and vary the number of pseudotransmitters to show that pseudo-transmitters add instability to the system in the presence of external error, here it is a 10 foot range measurement error.

\section{Implementation results}

A representative example of the results from our 802.11 implementation is shown in Fig. 7. All data was collected in an office building where walls and metal filing cabinets create plenty of signal reverberation. $802.11 \mathrm{~b}$ in this environment gave too little variation in our $\mu$ s measurement resolution to be useful. 802.11a however provided large error, but with enough variation between range measurements to be usefully incorporated into a position estimate. Range measurement error using 802.11 a varied up to $60 \%$ of the total distance, yet a position estimate could still be provided which was within 20 feet of the real position. An example of this is shown in Fig. 6. The ability to predict position with such a high range measurement error is due to signal filtering in combination with the powerful position estimation 
algorithm developed in [7], and tested extensively in [5]. The algorithm can handle large measurement errors as long as additional measurements are introduced.

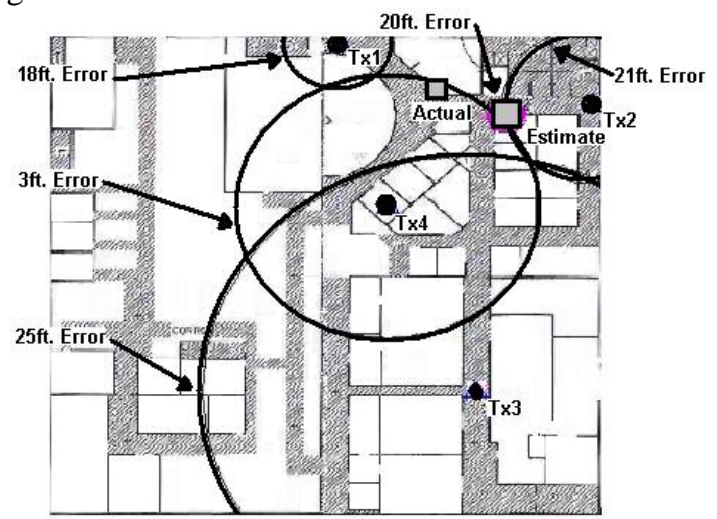

Figure 7. The results from the implementation show that a meaningful position estimate can still be calculated with $50 \%$ range measurement error.

\section{CONCLUSION}

Our research has been successful in not only uncovering answers to our initial questions, but also laying the foundation necessary to implement our algorithms using recently available UWB radio hardware. Our MATLAB software package runs smoothly and is easy to use. We have tested thousands of random networks without algorithm error, and data collected from these tests has led to interesting insights. Four transmitting nodes in a network, rather than three, considerably improve the position estimate of a receiver. When operating with a $\pm 10 \mathrm{ft}$ range measurement error they average a position estimate accurate to within $3 \mathrm{ft}$. Above four, however, there is little improvement. Using receivers as pseudo-transmitters does not improve the position estimate for other receivers, as originally predicted. We have also quantified these dependencies.

\section{ACKNOWLEDGMENT}

This research was supported through an internal Engineering Technology Development program, Lawrence Livermore National Laboratory. The authors would like to thank Steve Azevedo, Deputy Division Leader for the EETD, for his support of the project. This work was performed under the auspices of the U.S. Department of Energy by University of California, Lawrence Livermore National Laboratory under Contract W-7405-Eng-48.

\section{REFERENCES}

[1] N. Bulusu, J.Heidemann, and D. Estrin, "GPS-less low cost outdoor localization for very small devices," IEEE Personal Communications Magazine, vol. 7, no. 5, pp. 28-34.
[2] F. Dowla, A. Spiridon, D. Benzel, T. Rosenbury. "Ultra-wideband Communication," FY02 Engineering technology Reports, Vol. 2, Laboratory Directed Research and Development, UCRL-53868-02, pp. 4, 2003.

[3] D. Estrin, L.Gilrod, G. Potie, and M. Srivastava, "Instrumenting the world with wirelesss sensor networks," in Proc. IEEE Int. Conf. Acoustics, Speech, Signal Processing, vol. 4, pp. 2033-2036, Salt Lake City, Utah, USA May 2001.

[4] C. Kent, P. Atwal, W. Lennon, F. Dowla, "Position Estimation of Access Points in 802.11 Wireless Networks," Technical Report UCRL-JRNL-201930, Lawrence Livermore National Laboratory, October 2003.

[5] C. Kent, F. Dowla, "Position Estimation of Transceivers in Communication Networks," Technical Report UCRL-JRNL-201075, Lawrence Livermore National Laboratory, October 2003.

[6] M, Skolnok, "Introduction to Radar Systems," McGraw-Hill Book Co., 1962, pp. 468-470.

[7] J. Smith and J. Abel, "Closed-Form Least-Squares Source Location Estimation from Range-Difference Measurements," IEEE Transactions on acoustics and Speech, vol. ASSP-35, no. 12, pp. 1661-1669, 1987.

[8] R.F. Stengel, Optimal Control and Estimation (Dover Publications, New York, 1994).

[9] M. Uchida, et., al., "A Vehicle-to-Vehicle Communication and Ranging System Based on Spread Spectrum Technique," IEEE Vehicle Navigation \& Information Systems Conference Proceedings, 1994. 